\title{
Frequency selection by soliton excitation in nondegenerate intracavity downconversion
}

\author{
Dmitry V. Skryabin ${ }^{1}$, Alan R. Champneys ${ }^{2}$, and William J. Firth ${ }^{1}$ \\ ${ }^{1}$ Department of Physics and Applied Physics, University of Strathclyde, Glasgow, G4 ONG, Scotland \\ ${ }^{2}$ Department of Engineering Mathematics, University of Bristol, Bristol, BS8 1TR, England
}

(June 14, 1999)

\begin{abstract}
We show that soliton excitation in intracavity downconversion naturally selects a strictly defined frequency difference between the signal and idler fields. In particular, this phenomenon implies that if the signal has smaller losses than the idler then its frequency is pulled away from the cavity resonance and the idler frequency is pulled towards the resonance and vice versa. The frequency selection is shown to be closely linked with the relative energy balance between the idler and signal fields.
\end{abstract}

Exchange of ideas between nonlinear optics and nonlinear dynamics of spatially distributed systems has produced a series of interesting results over the last decade, and has opened up one of the most active areas of current research. In this particular work we will consider novel phenomena associated with soliton excitation in the practically and fundamentally important area of nondegenerate intracavity down-conversion.

The essence of parametric down-conversion is virtual absorption of a pump photon at frequency $\omega_{p}$ with a subsequent reemission of two photons with frequencies $\omega_{i}$ and $\omega_{s}$, where indices $i$ and $s$ stand, respectively, for the idler and signal fields. Down-conversion can be realized both in free propagation and in intracavity schemes. The latter takes advantage of positive feedback provided by the mirrors and thus transforms the passive free propagation scheme into an active generator or optical parametric oscillator (OPO) [1,2].

Influence of the transverse degrees of freedom on the quantum [3] and classical [3 11] aspects of the parametric processes have recently become a subject of significant activity. Among the main attraction points on the classical side are localised structures [5-11]. Bright diffractionless localised excitations inside an optical cavity supported by different nonlinearities (cavity solitons) have been recently observed experimentally [12] and suggested for all-optical processing and storage of information [9.13], see also recent reviews 14. The large quadratic nonlinearities of artificially phase matched materials [2] make parametric cavity solitons [5 11] particularly attractive for practical application, especially where fast material response is an issue.

Down-conversion processes can be divided into degenerate and nondegenerate. In the former case idler and signal photons are identical while in the latter they differ in frequencies and/or polarizations. It has been shown that transverse patterns in nondegenerate OPO [4.5] and soliton dynamics in nondegenerate free propagation 11 have qualitative differences from their degenerate counterparts. The difference can be formally identified as due to an additional symmetry, in the differential phase of signal and idler fields 11]. This symmetry is suppressed in the degenerate case. As a result the frequency of the signal component of any solution, including solitons, is exactly half the pump frequency, $\omega_{s} \equiv \omega_{i}=\omega_{p} / 2$. On the other hand, in the nondegenerate case any arbitrary frequency difference $2 \Omega$ between the idler and signal fields still satisfies the condition $\omega_{p}=\omega_{s}+\omega_{i}$. This raises the question of whether there are any physical constraints on $\Omega$.

While in free propagation the value of $\Omega$ is limited only by phase matching conditions, this problem becomes more subtle in the OPO because cavity effects come into play. A review of early works on this issue, exploring approaches based on the plane wave approximation, can be found in [1]. More recently Longhi [1] has shown that if diffraction is included then $\Omega$ becomes a function of the magnitude of the transverse component of the signal and idler wave vectors, $\vec{k}_{\perp}^{i}=-\vec{k}_{\perp}^{s}$, of the exact travelling wave solution which exists in this system [4]. For the fixed OPO parameters $\left|k_{\perp}^{s, i}\right|$ can take any values from certain continuous bands and hence so can $\Omega$.

The primary object of this Letter is to demonstrate that $\Omega$ is constrained when a cavity soliton is excited in the nondegenerate OPO. We show how this follows from a general relation between the soliton energies and the cavity losses. Existence of this relation, which has not been previously identified in this context, seems to be closely related to survival of differential phase symmetry in the presence of cavity losses. We use this symmetry to derive aproximate formulae for $\Omega$ in certain limits. Understanding this problem is not only practically important, but also holds the key to construction of entire families of cavity solitons.

Mean-field equations describing interaction of the signal, idler and pump waves in OPO [3, 1, 8] can be presented in the dimensionless quasi-Hamiltonian form

$$
\left(\partial_{t}+\gamma_{m}\right) E_{m}=i \frac{\delta H}{\delta E_{m}^{*}}, m=s, i, p
$$

where $H$ is the following functional: $H=$ $\int d x\left[-\alpha_{s}\left|\partial_{x} E_{s}\right|^{2}-\alpha_{i}\left|\partial_{x} E_{i}\right|^{2}-\alpha_{p}\left|\partial_{x} E_{p}\right|^{2}+\delta_{s}\left|E_{s}\right|^{2}+\right.$ $\delta_{i}\left|E_{i}\right|^{2}+\delta_{p}\left|E_{p}\right|^{2}+\left(E_{p} E_{i}^{*} E_{s}^{*}+\mu E_{s}^{*} E_{i}^{*}+\right.$ c.c. $\left.)\right] . \quad t$ is the time measured in the units of $\tau_{c} b$, where $\tau_{c}$ is the cavity round-trip time and $b$ is an arbitrary scaling constant. $\gamma_{m}=T_{m} b / 2$, where $T_{m}$ are the effec- 
tive mirror transmitivities. $\quad x=X\left[2 k_{s} /(b L)\right]^{1 / 2}$ and $\alpha_{m}=k_{s} / k_{m}$, where $X$ is the transverse coordinate in physical units, $k_{m}$ are the longitudinal components of the wave vectors and $L$ is a cavity round-trip length. $\delta_{m}=b \tau_{c}\left(\omega_{m}-\omega_{m}^{c a v}\right)$ are the detunings from the cavity resonances $\omega_{m}^{c a v}$. Physically, validity of these equations requires small losses and detunings: $\gamma_{m}, \delta_{m}<<b \pi$. Assuming, for simplicity, that $\omega_{s}$ is close to $\omega_{i}$, we can fix $\alpha_{s, i}=2 \alpha_{p}=1$, but still allow differences in $\delta_{s}, \delta_{i}$ and $\gamma_{s}, \gamma_{i}$. Then the phase matching conditions imply $n\left(\omega_{s}\right) \simeq n\left(\omega_{i}\right) \simeq n\left(\omega_{p}\right)$, where $n(\omega)$ is the linear refractive index and the following scalings can be derived for the field variables. $\mu$ is proportional to the external pump field $E_{p}: E_{p}=\mu \sqrt{2\left(\delta_{p}^{2}+\gamma_{p}^{2}\right) / \tilde{T}_{\omega_{p}}} /\left(b \tau_{c} \chi \omega_{s}\right)$, here $\chi$ is the effective quadratic susceptibility. The physical fields $\mathcal{E}_{m}$ are given by $\mathcal{E}_{s, i}=\sqrt{2} E_{s, i} e^{i \phi / 2} /\left(b \tau_{c} \chi \omega_{s}\right)$, $\mathcal{E}_{p}=2 e^{i \phi}\left(E_{p}+\mu \sqrt{\delta_{p}^{2}+\gamma_{p}^{2}}\right) /\left(b \tau_{c} \chi \omega_{s}\right)$, where $\phi=$ $-\operatorname{atan}\left(\gamma_{p} / \delta_{p}\right)$.

We seek localized solutions of Eqs. (1) in the form $E_{m}(x, t)=A_{m}(x, \Delta) e^{i \Omega_{m} t}$, where $\Omega_{s, i}= \pm \Delta \pm\left(\delta_{s}-\right.$ $\left.\delta_{i}\right) / 2, \Omega_{p}=0$. Then $A_{m}$ obey the set of differential equations

$$
\begin{aligned}
& -i \gamma_{s} A_{s}=\left(\partial_{x}^{2}+\delta-\Delta\right) A_{s}+\left(A_{p}+\mu\right) A_{i}^{*}, \\
& -i \gamma_{i} A_{i}=\left(\partial_{x}^{2}+\delta+\Delta\right) A_{i}+\left(A_{p}+\mu\right) A_{s}^{*}, \\
& -i 2 \gamma_{p} A_{p}=\left(\partial_{x}^{2}+2 \delta_{p}\right) A_{p}+2 A_{s} A_{i},
\end{aligned}
$$

where $\delta=\left(\delta_{s}+\delta_{i}\right) / 2$. The previously introduced frequency difference $2 \Omega$ between the idler and signal fields is linked with the parameter $\Delta$ by the formula: $2 \Omega=\left(2 \Delta+\delta_{s}-\delta_{i}\right) /\left(b \tau_{c}\right)$ We are interested in bright single-hump solitons, implying $A_{m}(x=+\infty)=0$ and $\partial_{x} A_{m}(x=0)=0$. Existence of such solitons in the parameter region where the trivial zero solution bifurcates subcritically can be predicted for $\Delta=0, \gamma_{s}=\gamma_{i}$ by analogy with the well studied degenerate case, where this condition reads $\delta \delta_{p}>\gamma_{s} \gamma_{p}$ [6,96]. In the nondegenerate case solitons have been found as a result of direct numerical simulations of Eqs. (1i) starting from either 'random' [5] or localized [8] initial conditions. Subcritical bifurcation and the related phenomenon of optical bistability in the nondegenerate OPO have been demonstrated experimentally in [15].

For fixed cavity parameters solitons can exist either for $\Delta$ continuously varying in a certain range or for only a discrete set of $\Delta$ values. We will show that the latter situation is realised for $\gamma_{s, i} \neq 0$. We thus assert that the cavity selects the frequency difference between the signal and idler when a parametric soliton is excited. Note that the related problem of frequency selection has been studied in the general context of the complex Ginzburg-Landau equation (CGLE) with subcritical bifurcation 16], which also can be applied to describe laser with saturable absorber [12,17]. The possibility of the approximate reduction of Eqs. (1) to the CGLE in the different limits has been demonstrated in [8]. However, the problem of the frequency selection by solitons in OPO has not been previously formulated even within the framework of the CGLE approximation. This fact has in turn prevented construction of the family of stationary soliton solutions of Eqs. (2) and rigorous study of their stability.

To start our analysis of the frequency selection, problem, we define the energy parameters $Q_{m}=\int d x\left|E_{m}\right|^{2}$ and the energy imbalance $\left(Q_{-}=Q_{s}-Q_{i}\right)$. Manipulation of Eqs. (1) reveals that the rate of change of $Q_{-}$is given by

$$
\partial_{t} Q_{-}=-2 \gamma_{+} Q_{-}-2 \gamma_{-} Q_{+}=2 \gamma_{i} Q_{i}-2 \gamma_{s} Q_{s},
$$

here $\gamma_{ \pm}=\left(\gamma_{s} \pm \gamma_{i}\right) / 2$ and $Q_{+}=Q_{s}+Q_{i}$. Thus for any steady state solution, such as soliton solutions of Eqs. (2), the condition

$$
\gamma_{s} Q_{s}=\gamma_{i} Q_{i},
$$

must be satisfied. Eq. (何) is consistent with the expectation that the field with the smaller losses will have the larger energy.

To further interpret relations (3), (4) let us recall that in free propagation downconversion is a Hamiltonian process. Then by Noether's theorem every continuous symmetry implies a corresponding conservation law, see e.g. 11. Cavity losses destroy the Hamiltonian structure of the problem, see Eqs. (1), and the input pump breaks the phase symmetry linked with conservation of the total energy $\left(Q_{s}+Q_{i}+2 Q_{p}\right)$. The symmetry $\left(E_{s}, E_{i}\right) \rightarrow\left(E_{s} e^{i \psi}, E_{i} e^{-i \psi}\right)$ in the differential phase $\psi$, however, survives in the cavity configuration, so how do the losses change the associated law $\partial_{t} Q_{-}=0$ ? Selfevidently the relation (3) becomes this conservation law in the Hamiltonian limit, which suggests a more general link between this energy relation and the symmetry in the differential phase.

Now using cavity solitons as an example we will demonstrate that condition (4) constrains the frequency difference of the signal and idler components of the intracavity field. We consider signal and idler losses small compare to the cavity detunings $\gamma_{s, i} /|\delta| \ll 1$ and set $b=1$. Conservation of $Q_{-}$is restored for $\gamma_{s, i}=0$ and thus Eq. (4) is satisfied automatically. A soliton family then exists for $\delta<0$, with $\Delta$ continuously varying in the interval $(\delta,-\delta)$. Outside this interval exponential localization of $A_{s, i}$ is not possible. Now suppose that for $\gamma_{s, i} \sim \epsilon \ll 1$ $\Delta$ becomes a slow function of $t$, i.e. $\partial_{t} \Delta \sim \epsilon$, then Eq. (3) immediately gives an equation for the adiabatic evolution of $\Delta: \partial_{t} \Delta \cdot \partial_{\Delta} Q_{-}=-\gamma_{+} Q_{-}(\Delta)-\gamma_{-} Q_{+}(\Delta)$. For stationary soliton solutions $\partial_{t} \Delta=0$ and intersections of the curve $Q_{s} / Q_{i}$ as a function of $\Delta$ with the line $Q_{s} / Q_{i}=\gamma_{i} / \gamma_{s}$ give selected values of $\Delta$. We plot in Fig. 1(a) the existence curve corresponding to the numerically built [18] single-hump soliton family in the plane $(\mu, \Delta)$ 
for $\delta=-1$ and losses of the signal and idler of order several percent. Dots in Fig. 1(a) correspond to values of $\Delta$ obtained by the perturbative method. The agreement is excellent, which also indicates that the limit of small cavity losses is non-singular. The latter is a necessary condition for a linkage between Eq. (3) and the differential phase symmetry. Typical transverse profiles of the soliton components are presented in Fig. 1(b).

To study stability of the solitons we seek solutions of Eqs. (1) in the form $\left(A_{m}(x)+\epsilon\left(u_{m}(x, t)+\right.\right.$ $\left.\left.i w_{m}(x, t)\right)\right) e^{i \Omega_{m} t}$. After standard linearization we derive $\partial_{t} \vec{\xi}=\hat{\mathcal{L}} \vec{\xi}$, where $\vec{\xi}=\left(u_{s}, u_{i}, u_{p}, w_{s}, w_{i}, w_{p}\right)^{T}$ and $\hat{\mathcal{L}}$ is the linear non-self-adjoint differential operator. The discrete spectrum of $\hat{\mathcal{L}}$ has been found numerically using second-order finite differences. Any discrete eigenvalue of $\hat{\mathcal{L}}$ with positive real part makes the soliton unstable. The new soliton family turns out to be stable over the section $(A, H)$ in Fig. 1(a). The Hopf instability of the $(H, B)$ branch, and the instability of the $(O, A)$ branch are similar to the case of the degenerate OPO [10]. At the point $B$ the single-hump branch bifurcates back in $\mu$, initiating a sequence of multi-hump solitons.

The above perturbation approach to find selected values of $\Delta$ requires $\gamma_{s, i}$ small, which is satisfied in most practical situations, but we also assumed $\delta \sim O(1)$. This fails if $\gamma_{s, i} /|\delta| \sim O(1)$ or $\sim \epsilon^{-1}$, which physically means that the cavity becomes tuned close to resonance with the signal and idler fields. Then terms proportional to $\delta$ in Eqs. (2) should also be considered as perturbations. In this case Eqs. (2) simply do not have solitary solutions in zero order and therefore $Q_{s, i}$ can't be considered functions of $\Delta$. To overcome this difficulty we used another perturbation approach, also based on the $\psi$-symmetry. Note first that the cavity solitons become wider on approach to resonance [19], and so to avoid large computational windows it is convenient to take large $b$, e.g. $b=2 / T_{s}$. Then $\gamma_{m} \sim 1$ physically corresponds to small losses. Now, observing that $A_{i}=A_{s}$ is a solution of Eqs. (2) for $\gamma_{s}=\gamma_{i}$ and $\Delta=0$, we assume $\gamma_{-},|\Delta| \sim \epsilon$. At first order in $\epsilon$

$$
\epsilon\left(\hat{\mathcal{L}}-\partial_{t}\right) \vec{\xi}=\Delta \vec{\xi}_{\psi}-\gamma_{-} \overrightarrow{\mathcal{P}},
$$

where $\vec{\xi}_{\psi}=\left(-\operatorname{Im} A_{s}, \operatorname{Im} A_{s}, 0, \operatorname{Re} A_{s},-\operatorname{Re} A_{s}, 0\right)^{T}$ is the neutral mode generated by the $\psi$-symmetry, i.e. $\hat{\mathcal{L}} \vec{\xi}_{\psi}=0$, and $\overrightarrow{\mathcal{P}}=\left(\operatorname{Re} A_{s},-\operatorname{Re} A_{s}, 0, \operatorname{Im} A_{s},-\operatorname{Im} A_{s}, 0\right)^{T}$. Eq. (5) immediately yields

$$
\Delta=\gamma-\frac{\left\langle\overrightarrow{\mathcal{P}} \mid \vec{\zeta}_{\psi}\right\rangle}{\left\langle\vec{\xi}_{\psi} \mid \vec{\zeta}_{\psi}\right\rangle},
$$

where the new vector $\vec{\zeta}_{\psi}$ is the neutral mode of $\hat{\mathcal{L}}^{\dagger}$, i.e. $\hat{\mathcal{L}}^{\dagger} \vec{\zeta}_{\psi}=0$, generated by the $\psi$-symmetry (which can be found numerically). As usual, $\langle. . \mid .$.$\rangle defines inner product$ in $L_{2}$. We again find an excellent agreement between Eq. (6) and numerical solutions of Eqs. (2), see Fig. 2(a).
In this case, stability analysis reveals that the solitons are stable along the entire segment $(A, B)$, with $B$ again marking the transition to multi-hump soliton solutions. We also found that $\left\langle\overrightarrow{\mathcal{P}} \mid \vec{\zeta}_{\psi}\right\rangle /\left\langle\vec{\xi}_{\psi} \mid \vec{\zeta}_{\psi}\right\rangle$ is positive throughout a wide region of parameters. This implies that the sign of $\gamma_{-}$determines the sign of $\Delta$, at least for parameter values where our perturbative approach is valid. Fig. 1(a) and selective numerical checks in parameter regions far outwith the validity of our perturbative methods support a conjecture that the relationship $\operatorname{sgn} \gamma_{-}=\operatorname{sgn} \Delta$ has a general character. Note finally that any difference in diffraction constants $\alpha_{s}$ and $\alpha_{i}$ will also affect the frequency selection, but we leave this mechanism for future analysis.

To assess possibilities of experimental observation of these cavity solitons let us take as an example a $1 \mathrm{~cm}$ long monolithic planar waveguide cavity with $\chi^{(2)} \simeq 20 \mathrm{pm} / V$, typical for a noncritically phase matched potassium niobate crystal. Suppose the waveguide to be $\sim 1 \mathrm{~mm}$ wide and $\sim 1 \mu m$ thick, excited by an elliptical pump beam at frequency $\sim 10^{15} \mathrm{~Hz}$. Other parameters as for Fig. 2(a) then imply the following real world values: pump power $\sim \mu^{2} \times 1 W$, selected frequency difference $\sim \Omega \times 10^{9} \mathrm{~Hz}$ and cavity soliton width $\sim 30 \mu \mathrm{m}$.

To excite cavity solitons one can apply spatially localised optical pulses at any of the three frequencies. Optimisation of the pulse parameters goes beyond of our present scope. However, to demostrate how the selection of the frequency difference happens, we show in Fig. 3 results of direct simulation of Eqs. (11) with, as initial condition, a pulse at frequency $\omega_{s}$ with duration around $0.1 \tau_{c}$, intensity several times the peak soliton intensity, and width of order the soliton width. Intensities of all three components of the excited soliton become constant after a transient period, see Fig. 3(a), while the real parts of the signal and idler fields exhibit undamped oscillations, confirming selection of $\Delta$ with the predicted value, see Fig. 3(b).

The main physical conclusion which can be drawn from the above results is that, if the signal has the smaller losses its frequency is pulled away from the cavity resonance while the idler frequency is pulled towards resonance and vice versa, see Figs. 1(a),2(a). If the signal and idler losses are equal then the selected value of $\Delta$ is zero, see Eq. (6), which implies that both the idler and the signal are equally detuned from cavity resonance. Thus the cavity structure balances the energies of the idler and signal components during soliton excitation, in accord with Eqs. (3), (4). The understanding of this frequency selection mechanism has allowed us to reconstruct an entire family of single-hump cavity solitons and to study their stability. Our results are likely to find applications in interpretation of other spatio-temporal phenomena in nondegenerate OPOs and also to be relevant in other intracavity parametric processes with symmetry in the differential phase, e.g., second harmonic generation 
with competing parametric process [20] and nondegenerate four-wave mixing 21.

We are indepted to G.K. Harkness, D. Michaelis and U. Peschel for assistance with numerical problems at the early stage of the work and to G.J. de Valcárcel for insightful remarks. D.V.S. acknowledges financial support from the Royal Society of Edinburgh and British Petroleum. The work is partially supported by ESPRIT project PIANOS and EPSRC grant GR/M19727.

[1] C. Fabre, in "Advanced photonics with second-order optically nonlinear processes", eds. A.D. Boardman et al. (Kluwer, Dordrecht, 1998), pp. 293-318.

[2] V. Berger, see [1], pp. 345-374.

[3] L.A. Lugiato and G.L. Oppo, Optics Express 3, 60 (1998).

[4] S. Longhi, Phys. Rev. A 53, 4488 (1996).

[5] V.J. Sanchez-Morcillo et al., Phys. Rev. A 56, 3237 (1997).

[6] K. Staliunas and V.J. Sánchez-Morcillo, Opt. Commun. 139, 306 (1997); S. Longhi, Phys. Scripta 56, 611 (1997); S. Trillo and M. Haelterman, Opt. Lett. 23, 1514 (1998).

[7] M. Tlidi, P. Mandel, and M. Haelterman, Phys. Rev. E 56, 6524 (1997); M. Tlidi, P. Mandel, and R. Lefever, Phys. Rev. Lett. 81, 979 (1998); K. Staliunas, Phys. Rev. Lett. 81, 81 (1998); S. Trillo, M. Haelterman, and A. Sheppard, Opt. Lett. 22, 970 (1997); G.L. Oppo, A.J. Scroggie, and W.J. Firth, J. Opt. B 1, 133 (1999); M. Le Berre et al., J. Opt. B 1, 153 (1999).

[8] S. Longhi, Opt. Commun. 149, 335 (1998); P.S. Jian et al., Opt. Lett. 24, 400 (1999).

[9] D.V. Skryabin and W.J. Firth, Opt. Lett. 24, 1056 (1999).

[10] D.V. Skryabin, Phys. Rev. E 60, R3508 (1999).

[11] D.V. Skryabin and W.J. Firth, Phys. Rev. Lett. 81, 3379 (1998); A.V. Buryak, Y.S. Kivshar, and S. Trillo, Phys. Rev. Lett. 77, 5210 (1996).

[12] V.B. Taranenko, K. Staliunas, and C.O. Weiss, Phys. Rev. Lett. 81, 2236 (1998); K. Staliunas et al., Phys. Rev. A 57, 599 (1998).

[13] W.J. Firth and A.J. Scroggie, Phys. Rev. Lett. 76, 1623 (1996); W.J. Firth, A. Lord, and A.J. Scroggie, Phys. Scripta T67, 12 (1996); M. Brambilla et al., Phys. Rev. Lett. 79, 2042 (1997); L. Spinelli et al., Phys. Rev. A 58, 2542 (1998); D. Michaelis, U. Peschel, and F. Lederer, Opt. Lett. 23, 337 (1998).

[14] N.N. Rosanov, Prog. Opt. 35, 1 (1996); W.J. Firth and G.K. Harkness, Asian J. Phys. 7, 665 (1998).

[15] C. Richy et al., J. Opt. Soc. Am. B 12, 456 (1995).

[16] B.A. Malomed, Physica D 29, 155 (1987); S. Fauve and O. Thual, Phys. Rev. Lett. 64, 282 (1990).

[17] T. Kapitula and B. Sandstede, J. Opt. Soc. Am. B 15, 2757 (1998); A.G. Vladimirov et al., J. Opt. B 1, 101 (1999).

[18] E.J. Doedel, A.R. Champneys, T.R. Fairgrieve, Y.A.
Kuznetsov, B. Sandstede, and W. Wang, AUTO97 Continuation and Bifurcation Software for Odinary Differential Equations, 199\%. Available from ftp.cs.concordia directory pub/doede/auto.

[19] D.V. Skryabin, in Nonlinear Guided Waves and Their Applications 1999, p. 154, OSA Technical Digest Series (OSA Washington DC, 1999).

[20] M.A.M. Marte, Phys. Rev. A 49, R3166 (1994); P. Lodahl and M. Saffman, Phys. Rev. A 60, 3251 (1999).

[21] R.E. Slusher et al., Phys. Rev. Lett. 55, 2409 (1985).
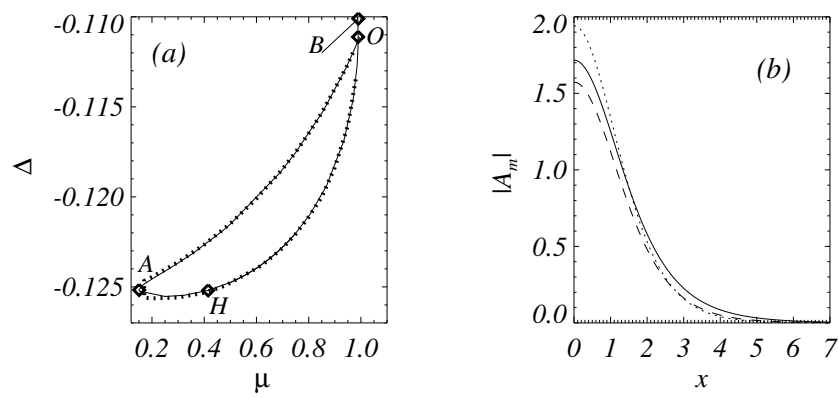

FIG. 1. (a) Existence curve in the $(\mu, \Delta)$-plane for the family of single-hump cavity solitons, demonstrating selection of $\Delta$. Full line corresponds to the results of numerical continuation, dotted line was obtained using perturbative methods (see text) in the limit $\gamma_{s, i} /|\delta| \ll 1: \delta=\delta_{p}=-1, \gamma_{s}=0.04$, $\gamma_{i}=0.05, \gamma_{p}=0.1, b=1$. Solitons are stable only in the small interval $(A, H)$, see text for details. (b) Soliton transverse profile for $\mu=0.3, \Delta=-0.1255007$. Full line: $\left|A_{s}\right|$, dashed line: $\left|A_{i}\right|$, dotted line: $\left|A_{p}\right|$.
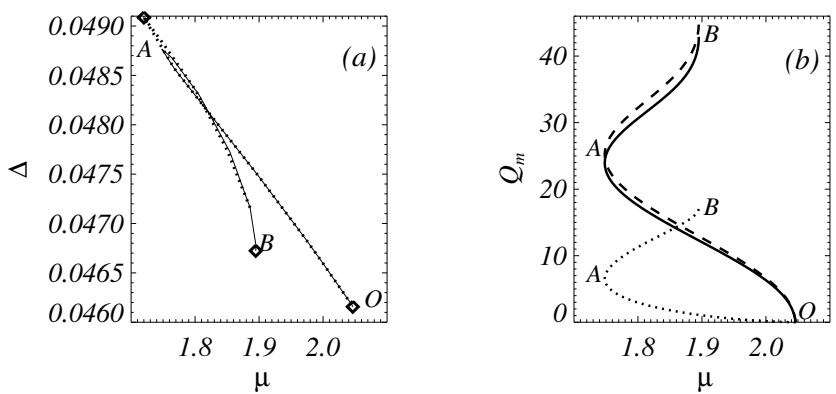

FIG. 2. (a) Existence curve in the $(\mu, \Delta)$-plane for family of single-hump cavity solitons, demonstrating selection of $\Delta$. Full line corresponds to the results of numerical continuation, dotted line was obtained using Eq. (6): $\delta=-1.8, \delta_{p}=-4$, $\gamma_{s}=1, \gamma_{i}=0.95, \gamma_{p}=2, b=2 / T_{s}$. Solitons are stable only in the interval $(A, B)$. (b) Plots showing dependence of $Q_{m}$ vs $\mu$. Parameters as for (a). Full line: $Q_{s}$, dashed line: $Q_{i}$, dotted line: $Q_{p}$. 

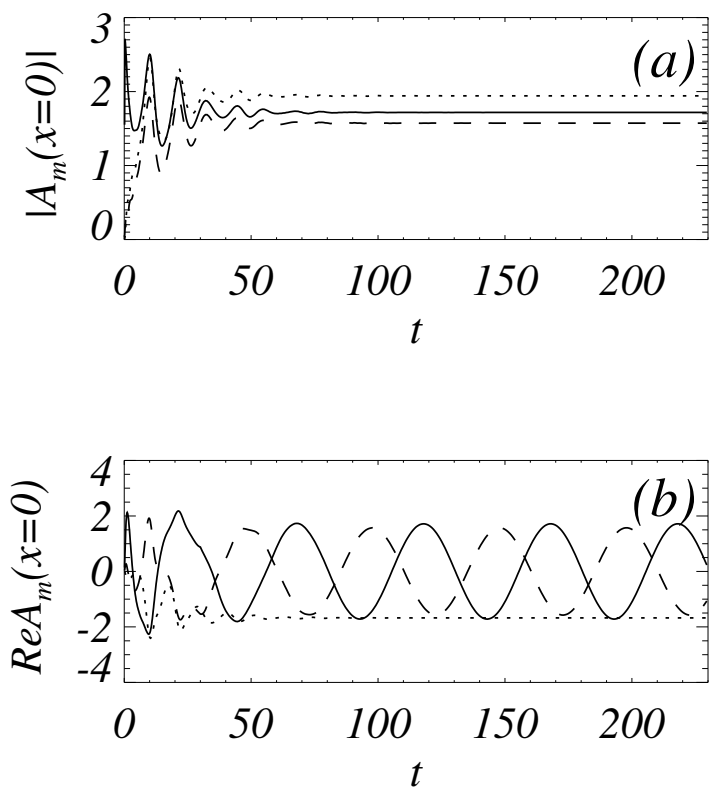

FIG. 3. Soliton excitation by a localized signal-field pulse. Temporal evolution of (a) $\left|E_{m}\right|$ and (b) $R e E_{m}$ at $x=0$. Full line: signal, dashed line: idler, dotted line: pump. Other parameters as for Fig. 1(b). Our predicted $\Delta$ corresponds to a period $\sim 50$ time units. 